\title{
Exotic viruses
}

\section{Arie Zuckerman offers this brief on some of the diseases now attracting broad attention}

\begin{abstract}
A BRIEF perusal of the daily press over the past few months would reveal an incidence of unusual disease which, to many, seems quite remarkable. There have, most recently, been the illnesses reported from the Sudan, Nigeria, Zaire and Sierra Leonenow apparently identified, by the World Health Organisation last week, as Marburg disease. Work on the identification of Philadelphia's so-called Legion's Disease continues in the United States. Lassa fever meanwhile attracts considerable attention. Rabies and Swine influenza receive even greater emphasis. Even Bubonic Plague has been in the headlines.

Of the many viruses that can infect more than one species of animal, three are of particular current interest. Rabies, with a huge reservoir of infection in the wildlife population of most parts of the world, is now racing across Europe towards Britain, where it has not been endemic for more than half a century. Marburg disease, first identified nine years ago, is still not fully understood. And Lassa fever looms as an awesome viral infection in tropical Africa.
\end{abstract}

\section{Marburg disease}

Marburg virus (or "green monkey") disease, a severe, distinctive, febrile human illness, was first described in 1967. Thirty-one cases of illness with seven deaths in Germany and Yugoslavia were traced to direct contact with blood, organs or tissue cell cultures from a batch of African green monkeys caught in Uganda. Several secondary cases subsequently occurred in hospital personnel who had been in contact with blood from patients; one further case was apparently transmitted by sexual intercourse. Although 29\% of the primary cases proved fatal, there were no deaths in the 6 secondary cases.

It soon became apparent that this was a previously unrecognised disease, caused by an infectious agent probably new to medical science. Laboratory work using guinea pigs and Rhesus and vervet monkeys revealed an elongated sinuous organism generally curled in the form of a figure 6 or a horseshoe with superficial resemblance to rabies virus. But Marburg virus is morphologically distinct and does not share any antigenic properties with rhabdoviruses or with any other known viruses.

The infection has been investigated intensively since the first outbreak, but the natural cycle of transmission in nonhuman primates and perhaps other species of animals and the origin of the infection remain unknown. The disease has not hitherto been recognised in a sporadic form in Africa.

The first recognised outbreak of the disease in Africa, and the first since the original outbreak, occurred in South Africa in February, 1975. The victim, a young Australian man who had hitch-hiked through Rhodesia, died in a Johannesburg hospital; shortly afterwards his travelling companion and one of the nurses who had cared for him contracted the same disease. Both recovered. Virological investigations showed the outbreak was caused by the Marburg virus. As in the original outbreak in Marburg, when the virus was isolated from semen 83 days after the onset of illness evidence was obtained that the virus could persist in the body for at least 2-3 months after the initial attack; the virus in this case was isolated from the victim's eye fluid 80 days after the onset of the illness.

\section{Lassa fever}

The dramatic story of Lassa fever began when two missionary nurses from Lassa, in North-Eastern Nigeria, died in 1969 from a mysterious illness; a third, who was gravely ill and flown to the United States, recovered. Convalescent plasma from her was effective in the treatment of a laboratory worker, who acquired the infection while working with tissue cultures infected with blood from these patients.
The following year a further outbreak of Lassa fever killed $52 \%$ of 23 patients admitted to hospital in Nigeria. Dr Jeannette Troup, who was primarily responsible for drawing attention to this disease, also died of the infection. Since then further cases have occurred and 10 out of 21 medical workers have died from Lassa fever. Up to June, 1975 the disease was recognised on nine different occasions involving 114 officially recorded cases, two of which resulted from laboratory infections. Over a third of these infections were acquired by person-toperson spread within hospitals. Many other clinically suspect cases have since been uncovered from reviews of hospital records: retrospective searches through the medical literature have revealed references to cases closely resembling Lassa fever in the Central African Republic, Upper Volta, Nigeria and Sierra Leone. Since 1969, cases have occurred in Nigeria, Sierra Leone and Liberia, and serological evidence suggests the virus may have been active elsewhere in West and Central Africa. And of course recent cases identified in air travellers to Britain, the United States and Canada have received extensive press coverage.

The clinical spectrum of Lassa fever extends from very mild or inapparent illness to fulminating fatal infection. Among patients admitted to hospital the death rate is as high as $67 \%$, with more fatalities among Caucasians than Asians. Intimate contact with any primary case appears to carry a high risk of infection; relatives or medical attendants providing direct personal or nursing care are particularly threatened. Cases have occurred among patients and hospital visitors who apparently had no direct contact with the primary cases. Only a few such tertiary infections have occurred, and the reasons for the interruption of

\section{Sorry, for copyright \\ reasons some images on this page may not be available online}


outbreaks after the generation of secondary cases are not clear. It seems that, although the primary cases were highly infectious, the secondary cases were not.

Lassa virus is a member of the arenavirus genus, a group of enveloped RNA viruses with a virion of granules instead of a defined core. Along with Machupo virus (which causes Bolivian haemorrhagic fever) it produces a significant mortality in man. Lassa appears to have the greatest potential for direct spreading in human populations. It is related antigenically to the rodent-associated haemorrhagic viruses of South America and to lymphocytic choriomeningitis virus, a persistent infection of mice. It has been isolated from a single rodent species, the multimammate rat Mastomys natalensis in West Africa.

More recently, a virus closely related immunologically to Lassa virus was recovered from the tissues and organs of wild Mastomys natalensis captured in 1972 in Mozambique. This finding more than doubles the potential area of Lassa-like virus distribution in Africa. The multimammate rat is the most widespread and commonest rodent in Africa south of the Sahara, and an ideal carrier to the domestic environment because of its semi-commensal habit and a combination of other factors, including an exceptionally high propagation rate. Whether all the Mastomys rodents are capable of maintaining and transmitting Lassa virus, is not known, and the ecological interrelationships between Mastomys and the presumed non-reservoir genera Rattus and Mus have yet to be determined. Carnivores, in particular dogs and cats which probably prey upon Mastomys and other rodents, have not yet been investigated as potential secondary hosts.

The Lassa virus may spill over from the rodent cycle to man by various routes including, for example, contamination with rodent urine, directly or on foodstuffs or through dust. The means by which the virus is spread from one person to another is not clear. Accidental inoculation with a sharp instrument or contact with blood has accounted for a few cases. The virus has been isolated from the pharynx and urine of patients, so that indirect airborne spread of the virus as well as mechanical transmission are most likely.

Lassa fever is not a disease which comes under international quarantine regulations, which at present offer the only effective means of reducing the risk of transmission. Transmission can be limited to some extent by surveillance, identification, and the hospitalisation and isolation of patients, but quarantine of households may have to be considered by public health authorities, and ways of reducing rodent populations should be explored. The evacuation of patients presents special problems. Until effective methods of treatment and control become available and a suitable vaccine is developed, the epidemiological propensities of this infection may be very wide with the modern means of air travel.

\section{Rabies}

Rabies virus is a member of the rhabdovirus group, which includes a diverse collection of characteristic bullet-shaped RNA viruses from mammals, reptiles, fish, insects and plants. The concept of a single antigenic entity is no longer accepted since there are four recognised rabiesrelated viruses, although these appear to be confined to sub-Saharan Africa. Two of these viruses, Mokola and Duvenhage, are associated with fatal rabies-like human illness.

Rabies is a viral infection of the central nervous system which has been recorded in most domesticated and wild warm-blooded animals. The virus, present in salivary secretions, is transmitted mainly by bites or scratches, although the inhalation of minute droplets in the air can also lead to infection, and oral transmission has been demonstrated in experimental animals. The susceptibility of an animal depends on its age, the degree of infection, the site of introduction and the properties of the particular virus strain involved. Once the virus leaves the site of inoculation it usually passes to the central nervous system through the peripheral nerves. Once the central nervous system has become infected the virus may multiply, attacking such tissues as the salivary and other glands, the kidneys, the brown fat, and, in some species, muscle and lung. In some infected organs the virus is found primarily within the nerves.

The effects of the disease on the nervous system and the change of behaviour in the victim are probably important to the mode of transmission. Typically, the sick animal (and this applies particularly to small carnivores) becomes unusually and indiscriminately aggresive, while in the earlier stages of infection timid animals become less restrained, approachable and friendly. High population density and turnover and long incubation periods maintain the infection even though it is usually fatal. Man is irrelevant to survival of the virus: there is no adequately documented case of human transmission of rabies, although a theoretical risk exists because the virus is present in secretions.

The most important pool of infec- tion in most areas of the world is the wildlife population, including, in particular, foxes. Examination of thousands of small wild rodents in the Americas has not revealed rabies infection, but in parts of Europe the isolation of rabies virus with unusually low virulence has been reported from wild rodents. The significance of this finding is not clear, and cannot be immediately associated with fox rabies. The ecology of bat rabies is intriguing: in the Americas, numerous species of bats have been found with rabies infections, but in many areas these infections are independent of other mammalian cycles. The disease is readily passed on to cattle by the bite of vampire bats during feeding; in Latin America, for example, bat rabies is estimated to have killed many hundreds of thousands of cattle. Over 170 human deaths from rabies have been attributed to vampire bats, which suck the blood of sleeping victims. But natural transmission from insectiverous bats to other terrestrial animals by biting has not been observed, and experimental transmission with infectious saliva has proved difficult.

The major problem caused by rabies, according to a paper from the Office of Health Economics in the UK, does not stem from its immediate effects, but from the medical, social and economic risks and the cost associated with its prevention. Improved human diploid cell vaccines are in fact now becoming available for pre- and postexposure prophylaxis in man, while supplies of human rabies immunoglobulin for passive immunisation and local wound treatment are expected to increase and developments in intensive care promise a better outlook for future victims.

The control of rabies in animals, on the other hand, presents considerable difficulties. The international transfer of animals must be rigidly controlled. and strict precautions should be taken in the acquisition of mammals from the wild. The control of wildlife rabies is even more difficult. Hormonal sterilisation can be effective experimentally but has not yet been used successfully in the field. The immunisation of wildlife may become feasible in the future, particularly oral immunisation of foxes using bait doped with live attenuated virus. The control of bat rabies requires further investigation, although inoculation of cattle with small amounts of anticoagulant has proved lethal for vampires. Similarly the backs of captured vampire bats can be smeared with anticoagulant mixed with petroleum jelly: their release has resulted in dramatic reduction in the number of bats, since the treated bats contaminated others in their colony by contact. 